\title{
FOUR-FERMION PRODUCTION THROUGH RESONATING BOSON PAIRS AT LEP2
}

\author{
Ramon Miquel \\ CERN, PPE Division, CH-1211 Geneva 23, Switzerland \\ Michael Schmitt* \\ Department of Physics, University of Wisconsin, Madison, WI 53706, USA
}

\begin{abstract}
A Monte Carlo program for the simulation of four-fermion final states at LEP2 is presented. It contains the Feynman diagrams with two resonating W's and Z's and features, among other things, initial and final state radiation, Coulomb singularity effects and effective couplings. It is interfaced to the JETSET package to handle gluon radiation, hadronisation and decays. Its usefulness for experimental studies is stressed. Comparisons with some other calculations show that it is precise enough for most experimental uses.
\end{abstract}

(Submitted to Zeitschrift für Physik C)

*Supported by the US Department of Energy, grant number DE-FG0295ER40896. 


\section{Introduction}

The second phase of the LEP $e^{+} e^{-}$collider will start routine operation above the W-pair production threshold in 1996. Experimental physics studies need Monte Carlo event generators that simulate accurately the expected physics distributions $[1,2,3]$.

We present a Monte Carlo event generator (LPWW) based on a complete Monte Carlo calculation of the cross section for the process $e^{+} e^{-} \rightarrow f_{1} \bar{f}_{2} f_{3} \bar{f}_{4}$ through a pair of resonating heavy bosons, WW and/or ZZ [4]. This calculation was done for off-mass-shell heavy bosons and included both full spin transmission from initial to final state and interference between WW and ZZ mediated amplitudes. The calculation is described in section 2.

Our goal has been to build a Monte Carlo program that could be readily used for the experimental studies needed at the preparation stage of LEP2 and for most of the studies when analysing LEP2 data. To achieve this, we have transformed the cross section calculation into an unweighted event generator and included higher order corrections: initial and final state radiation, dominant weak corrections through the use of effective couplings, Coulomb singularity effects, and running, QCD-corrected, $\mathrm{W}$ and $\mathrm{Z}$ widths. In this way the program has the precision needed for most LEP2 studies. Details are given in section 3 .

We have also interfaced the program with the JETSET [5] package to simulate gluon radiation, hadronisation of quarks and gluons and decays of hadrons and tau leptons, so that it can reproduce the observable final states. The final state flavours can be chosen at will or, alternatively, the expected flavour mix at each centre-of-mass energy can be automatically generated. Results and comparisons with existing calculations are presented in section 4 .

\section{Matrix Element Computation and Phase-Space In- tegration}

The matrix element for the four-fermion production process $e^{+} e^{-} \rightarrow f_{1} \bar{f}_{2} f_{3} \bar{f}_{4}$ has been computed using the helicity amplitude techniques described in detail in [6]. This technique works in the massless fermion limit, which is a good approximation at LEP2 energies, at least when (as here) no diagrams with photons in the t-channel are considered.

In the massless limit, helicity is strictly conserved along fermionic lines. We denote by $\lambda, \rho, \sigma$ the helicities in the three fermionic lines appearing in any of the Feynman diagrams under consideration (fig. 1$)$ and by $p_{i}(i=1, \ldots, 6)$, the four-momenta of the six external fermions. Depending on the helicity configuration, the numerator of the amplitude of the first diagram in fig. 1 will be either zero or proportional to the function

$$
\begin{aligned}
A\left(\lambda, \rho, \sigma ; p_{1}, p_{2}, p_{3}, p_{4}, p_{5}, p_{6}\right)= & {\left[\bar{u}_{\lambda}\left(p_{1}\right) \gamma^{\mu} u_{\lambda}\left(p_{2}\right)\right] } \\
& {\left[\bar{u}_{\rho}\left(p_{3}\right) \gamma_{\mu}\left(p_{1}+p_{2}+p_{3}\right) \gamma_{\nu} u_{\rho}\left(p_{4}\right)\right] . } \\
& {\left[\bar{u}_{\sigma}\left(p_{5}\right) \gamma^{\nu} u_{\sigma}\left(p_{6}\right)\right] }
\end{aligned}
$$


where, since we are working in the massless limit, we have dropped the distinction between particle and antiparticle. In the language of ref. [6], the function $A$ can be written as

$$
A(+,+,+; 1,2,3,4,5,6)=-4\langle 31\rangle^{*}\langle 46\rangle\left[\langle 51\rangle^{*}\langle 21\rangle+\langle 53\rangle^{*}\langle 23\rangle\right],
$$

for the helicity configuration $\lambda=\rho=\sigma=+$. The spinorial products $\langle j k\rangle$ are given in terms of the momentum components by

$$
\langle j k\rangle \equiv\left(p_{j}^{1}+i p_{j}^{2}\right)\left[\frac{p_{k}^{0}-p_{k}^{3}}{p_{j}^{0}-p_{j}^{3}}\right]^{1 / 2}-(k \leftrightarrow j) .
$$

All helicity combinations for all abelian diagrams can be expressed in terms of the function appearing in eq. (1), with different arguments and occasional conjugations and changes of sign. The numerators of the non-abelian diagrams can be expressed as sums or differences of two or three $A$ functions. Details of the actual implementation of this treatment in a similar Monte Carlo program can be found in ref. [2].

The phase-space generation follows the multichannel generator approach described, for instance, in ref. [7]. Events are generated alternatively with their kinematic characteristics mapped to reproduce either those of a WW-mediated event or those of a ZZ-mediated event. Furthermore, the final-state flavours are chosen according to an approximate a priori probability and a correction is included in the final weight of the event. In this way, the final sample has the correct flavour content of four-fermion events produced through two resonating W's or Z's and the resulting event generator is easier to use. At this level, Cabibbo mixing between the first two generations is incorporated. Mixing involving the third generation of quarks is neglected.

In all cases, the four-particle phase-space density, $d R_{4}$, is generated according to the kinematics of the production and subsequent decay of two heavy particles:

$$
d^{8} R_{4}=d^{2} R_{2}\left(s ; s_{1}, s_{2}\right) d^{2} R_{2}\left(s_{1} ; 0,0\right) d^{2} R_{2}\left(s_{2} ; 0,0\right) d s_{1} d s_{2},
$$

with

$$
\begin{aligned}
d^{2} R_{2}\left(s ; s_{1}, s_{2}\right) & =\frac{1}{8}\left(1-\frac{s_{1}+s_{2}}{s}\right)\left(1-\frac{4 s_{1} s_{2}}{\left(s-s_{1}-s_{2}\right)^{2}}\right)^{\frac{1}{2}} d \cos \theta d \phi \\
d^{2} R_{2}\left(s^{\prime} ; 0,0\right) & =\frac{1}{8} d \cos \theta_{f}^{*} d \phi_{f}^{*},
\end{aligned}
$$

where $s^{\prime}$ stands for $s_{1}$ or $s_{2}$, the invariant masses of the W's or Z's, and the * superscript denotes variables in the rest frames of the decaying heavy-bosons.

In order to compensate for the peaking structure of the matrix element squared, the eight variables entering eqs. (2),(3) are generated according to:

$$
\begin{aligned}
\kappa_{1} d \eta_{1} & =\frac{d s_{1}}{\left(s_{1}-M_{W}^{2}\right)^{2}+\Gamma_{W}^{2} M_{W}^{2}} \\
\kappa_{2} d \eta_{2} & =\frac{d s_{2}}{\left(s_{2}-M_{W}^{2}\right)^{2}+\Gamma_{W}^{2} M_{W}^{2}}
\end{aligned}
$$



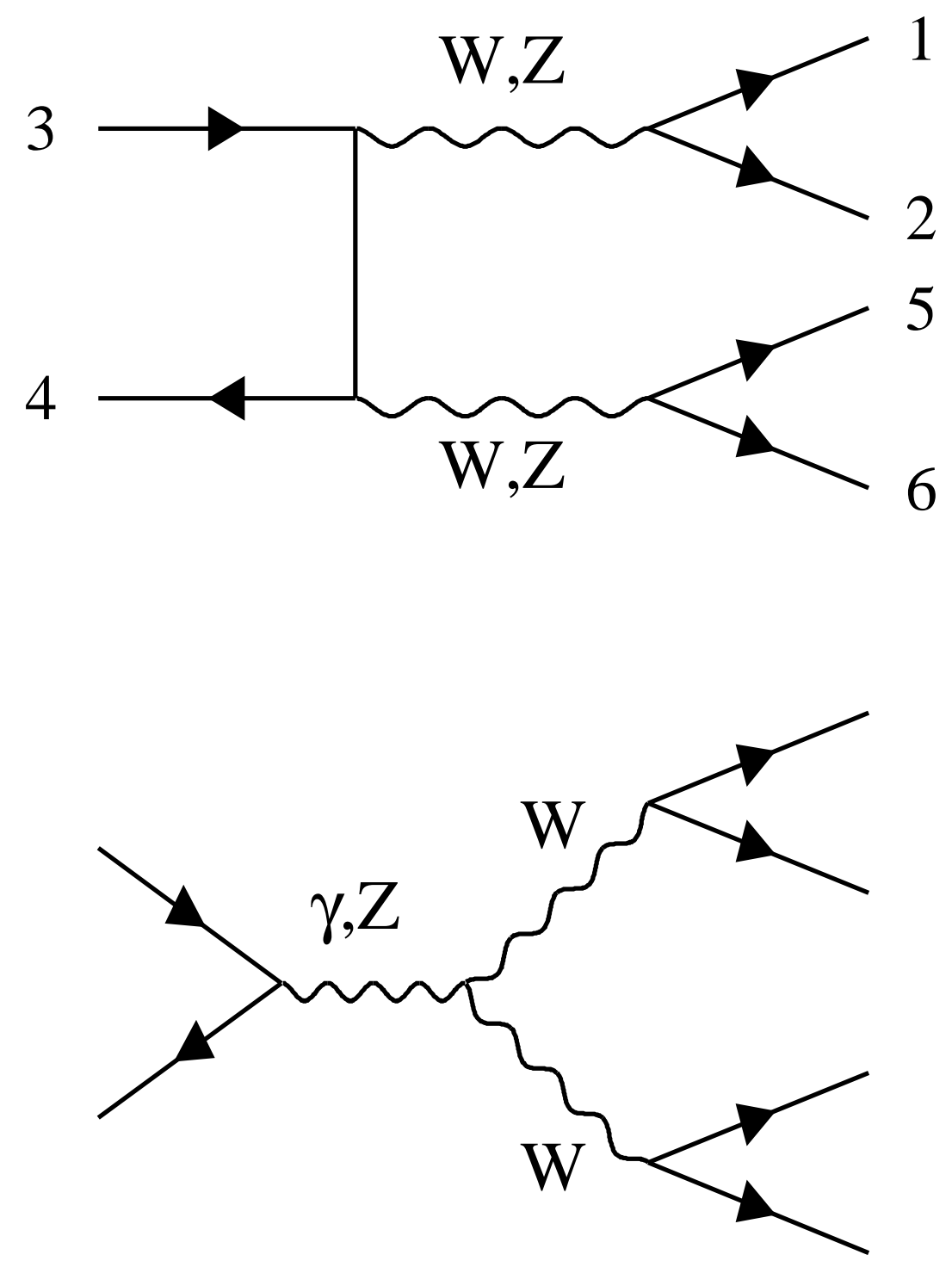

Figure 1: Feynman diagrams for the helicity amplitude calculation. 


$$
\begin{aligned}
\kappa_{3} d \eta_{3} & =\frac{d \cos \theta}{z\left(s, s_{1}, s_{2}\right)-\cos \theta} \\
\kappa_{4} d \eta_{4} & =d \phi \\
\kappa_{5} d \eta_{5} & =d \cos \theta_{1}^{*} \\
\kappa_{6} d \eta_{6} & =d \phi_{1}^{*} \\
\kappa_{7} d \eta_{7} & =d \cos \theta_{2}^{*} \\
\kappa_{8} d \eta_{8} & =d \phi_{2}^{*},
\end{aligned}
$$

where $\eta_{1}$ are random variables distributed uniformly between 0 and $1, \kappa_{1}$ are normalisation factors and the function $z$ reads

$$
z\left(s, s_{1}, s_{2}\right)=\left(1-\frac{s_{1}+s_{2}}{s}\right) /\left[\left(1-\frac{s_{1}+s_{2}}{s}\right)^{2}-\frac{4 s_{1} s_{2}}{s^{2}}\right]^{\frac{1}{2}}
$$

\section{Higher Order Corrections}

\subsection{Initial State Radiation}

Lacking a full $\mathcal{O}(\alpha)$ calculation including all virtual and real QED corrections to the fourfermion final state, we use the structure function approach for the initial state radiative (ISR) corrections and another leading-logarithmic method, described in the following section, for the corrections due to final state radiation (FSR).

The treatment of initial state radiation, based on the structure functions approach, has been widely used for LEP1 calculations [8, 9]. The Born-like cross section at the reduced centre-of-mass energy after initial-state radiation is convoluted with the structure functions of the electron and positron, which take into account their probabilities to radiate. The differential cross section after initial state radiation can be written as

$$
d^{8} \sigma(s)=\int_{z_{1}^{m}}^{1} d z_{1} \int_{z_{2}^{m}}^{1} d z_{2} D_{e}\left(z_{1}, s\right) D_{e}\left(z_{2}, s\right) d^{8} \sigma_{0}\left(s z_{1} z_{2}\right)
$$

where $z_{i}^{m}$ are the minimum allowed energies for the colliding electron and positron. The electron structure function, $D_{e}(z, s)$, taken from ref. [9], includes soft-photon exponentiation and leading-logarithmic corrections up to $\mathcal{O}\left(\alpha^{2}\right)$. It also includes some subleading corrections up to $\mathcal{O}\left(\alpha^{2}\right)$, which were computed for the process $e^{+} e^{-} \rightarrow \ell^{+} \ell^{-}$at LEP1. They are not exactly equal to the (uncalculated) second order subleading terms for the four-fermion process under consideration. The differences, however, are subdominant and, at this time, neglected.

Equation (4) is used in the collinear approximation and, hence, the photon direction is assumed to be that of the incoming beams. Consequently, no real photon four-momenta are generated inside the experimentally accessible regions of phase space. 
The radiation not only changes the effective centre-of-mass energy of the event, but also the centre-of-mass momentum with respect to the laboratory system. A boost is applied to the generated particles to take this into account.

The effects of initial-state radiation are large, especially close to threshold. At $\sqrt{s}=$ $170 \mathrm{GeV}$, for example, the cross section is decreased by $25 \%$ and the mean $W$ mass is shifted by about $+50 \mathrm{MeV}$. It should be noted that the effect in the measured $W$ mass is much larger (about $+400 \mathrm{MeV}$ ), if a constraint equating the total energy measured to the nominal centre-of-mass energy is used in the determination of the $W$ mass.

\subsection{Final State Radiation}

Photon radiation from final-state fermions is an important effect experimentally, especially for electrons and muons.

Radiation from quarks is taken care of by the JETSET package [5]. We employ the PHOTOS package [10] to simulate radiation from final state electrons and muons. Radiation from taus or their decay products is neglected. The algorithm in PHOTOS provides full kinematic information for the splitting $f \rightarrow f^{\prime} \gamma$. It is based on an implementation of $\mathcal{O}\left(\alpha^{2}\right)$ bremsstrahlung calculation in the leading-log approximation. This means that final state radiation does not influence the total cross section calculation in any way. The authors have checked explicitly the accuracy of their package in the case of $\mathrm{W} \rightarrow \mathrm{e} \nu(\gamma)$ by comparing to a specific calculation of this process [11]. At $\sqrt{s}=175 \mathrm{GeV}$, the mean number of FSR photons per event with energy exceeding one per cent of the fermion energy is 0.022 , and the mean energy of those photons is $2.0 \mathrm{GeV}$.

\subsection{Effective Couplings}

The bulk of the electroweak radiative corrections (excluding photon radiation) can be absorbed in the form of so-called "effective couplings", which render the corrected expressions similar to the Born-level ones. This is the so-called "Improved Born Approximation".

We follow the approach of ref. [12]. The only two coupling combinations appearing in the $\mathrm{W}$-pair production amplitudes are $g^{2}$ and $e^{2}$ for left-handed electrons and only $e^{2}$ for right-handed electrons. The substitutions

$$
g^{2} \rightarrow 4 \sqrt{2} G_{F} M_{W}^{2}, \quad e^{2} \rightarrow 4 \pi \alpha(s)
$$

account for the most important electroweak corrections [12]. Here, $G_{F}$ stands for the Fermi coupling constant and $\alpha(s)$ for the electromagnetic fine structure constant evaluated at the scale $s$.

The same substitutions are applied to the Z-pair production amplitudes. In this case the weak effective mixing angle, taken from the LEP1 measurements, is also needed.

The inclusion of these corrections increases the cross section in the LEP2 energy region by about $12 \%$, without affecting the mass, energy, or angular distributions. 


\subsection{Running Width}

The $W$ and $Z$ bosons propagators have been modified to include the mass dependence of their imaginary parts by substituting

$$
\frac{1}{\left(s_{i}-M_{W}^{2}\right)^{2}+\Gamma_{W}^{2} M_{W}^{2}} \rightarrow \frac{1}{\left(s_{i}-M_{W}^{2}\right)^{2}+\left(\frac{s_{i}}{M_{W}^{2}}\right)^{2} \Gamma_{W}^{2} M_{W}^{2}},
$$

and similarly for the $\mathrm{Z}$ propagator, in the evaluation of the matrix elements.

At $\sqrt{s}=170 \mathrm{GeV}$ this substitution decreases the mean $\mathrm{W}$ mass by about $20 \mathrm{MeV}$.

\subsection{QCD Corrections}

A final state QCD correction is included for the final states with hadrons by modifying the number of colours from $N_{C}=3$ to $N_{C}=3\left(1+\alpha_{s} / \pi\right)$.

This correction increases the total cross section by about $6 \%$ and changes the effective branching fractions of W's and Z's to hadrons and leptons.

No attempt has been made to address the issues of colour reconnection [13] or BoseEinstein correlations [14] at the level of the matrix element. They could be incorporated in the context of the JETSET hadronisation model. The interface with JETSET is described in section 4 .

\subsection{Coulomb Correction}

The radiative correction coming from the photon exchange between two non-relativistic charged particles (the so-called 'Coulomb singularity') can be a large effect close to the W production threshold. We have implemented the Coulomb correction in the production of two W's following ref. [15]. In this treatment, the correction to the cross section is given by $1+\delta_{C}$ with

$$
\delta_{C}=\frac{\pi \alpha}{2 \beta}\left(1-\frac{2}{\pi} \arctan \left(\frac{\left|\beta_{W}\right|^{2}-\beta^{2}}{2 \beta \operatorname{Im} \beta_{W}}\right)\right),
$$

where

$$
\begin{aligned}
\beta_{W} & =\left(1-\frac{4 \bar{M}_{W}^{2}}{s}\right)^{1 / 2} \\
\bar{M}_{W}^{2} & =M_{W}^{2}+i \Gamma_{W} M_{W} \\
\beta & =\frac{1}{s} \lambda^{1 / 2}\left(s, s_{1}, s_{2}\right),
\end{aligned}
$$

with $\lambda\left(s, s_{1}, s_{2}\right)$ the Mandelstam function and $s_{1}, s_{2}$ the invariant masses of the two (off-shell) W's.

The Coulomb correction increases the total cross section by as much as $4 \%$ at $\sqrt{s}=$ $165 \mathrm{GeV}$. 


\subsection{Hadronisation}

The most successful and widely-used model for hadronisation is the 'string' model implemented, for instance, in the JETSET package [5]. Given a colourless pair of quarks, it covers the processes of hard and soft gluon radiation (via parton showers), the formation of hadrons from partons, and the decay of resonances and unstable particles. It is a convenient and well-tested model, appropriate for the process $W \rightarrow$ hadrons.

The decays of the two W (or Z) bosons are independent, and in the case when both decay to quarks, we take their hadronisation also to be independent. Recently the possibility of colour interference (or 'reconnection') between the quarks from different W's has been discussed [13], but we have not tried to implement this in LPWW.

In order to allow for gluon radiation, hadronisation and decays, the kinematics of the event, massless up to this point, is recomputed using the correct fermion masses, taking care to respect conservation of energy and momentum.

The generated event consists essentially of the four-momenta of the final-state fermions. If the event could only be produced through a WW pair (or a ZZ pair), for instance when the final state is $u \bar{d} \bar{c}$, then the quarks coming from one $W$ (or Z) are defined as a colour-singlet, i.e., they are joined by a single colour string. Each colour-singlet is showered separately (e.g., first $\mathrm{u} \overline{\mathrm{d}} \rightarrow$ hadrons and then $\overline{\mathrm{c}} \mathrm{s} \rightarrow$ hadrons). A difficulty arises when the final state is consistent with both WW and ZZ intermediate states, such as u $\bar{d} \bar{u} d$. As explained in section 2, the peaking in phase space (either in $(u \bar{d})(\bar{u} d)$ or $(u \bar{u})(\bar{d} d)$ ) is simulated stochastically. The strings joining pairs of quarks are chosen to correspond to the choice made for the phase space generation.

\section{Results}

It is instructive to compare the results for the total cross section and for various physically interesting distributions, turning off and on the major corrections discussed in the previous section.

The total cross section depends strongly on initial-state radiative corrections, on the $\mathrm{W}$ width, and on the Coulomb singularity. We illustrate these effects in figure 2. Curve $a$ shows the Born cross section for bosons with no width. It is zero for $\sqrt{s}<2 M_{\mathrm{W}}$. The effect of the finite boson width is shown in curve $b$, which otherwise does not include radiative corrections or the Coulomb singularity. Initial-state radiation decreases the cross section by several percent as shown in curve $c$. Finally, the Coulomb attraction of the W's near threshold enhances the cross section by a couple of percent, as can be seen in curve $d$. This result was obtained for $M_{\mathrm{W}}=80.25 \mathrm{GeV}$ and $\Gamma_{\mathrm{W}}=2.088 \mathrm{GeV}$. Some representative values are listed in table 1.

The sharp falloff of the cross section at threshold suppresses events in which both bosons have a high mass. Since the exact shape of the falloff depends on initial-state radiation, 


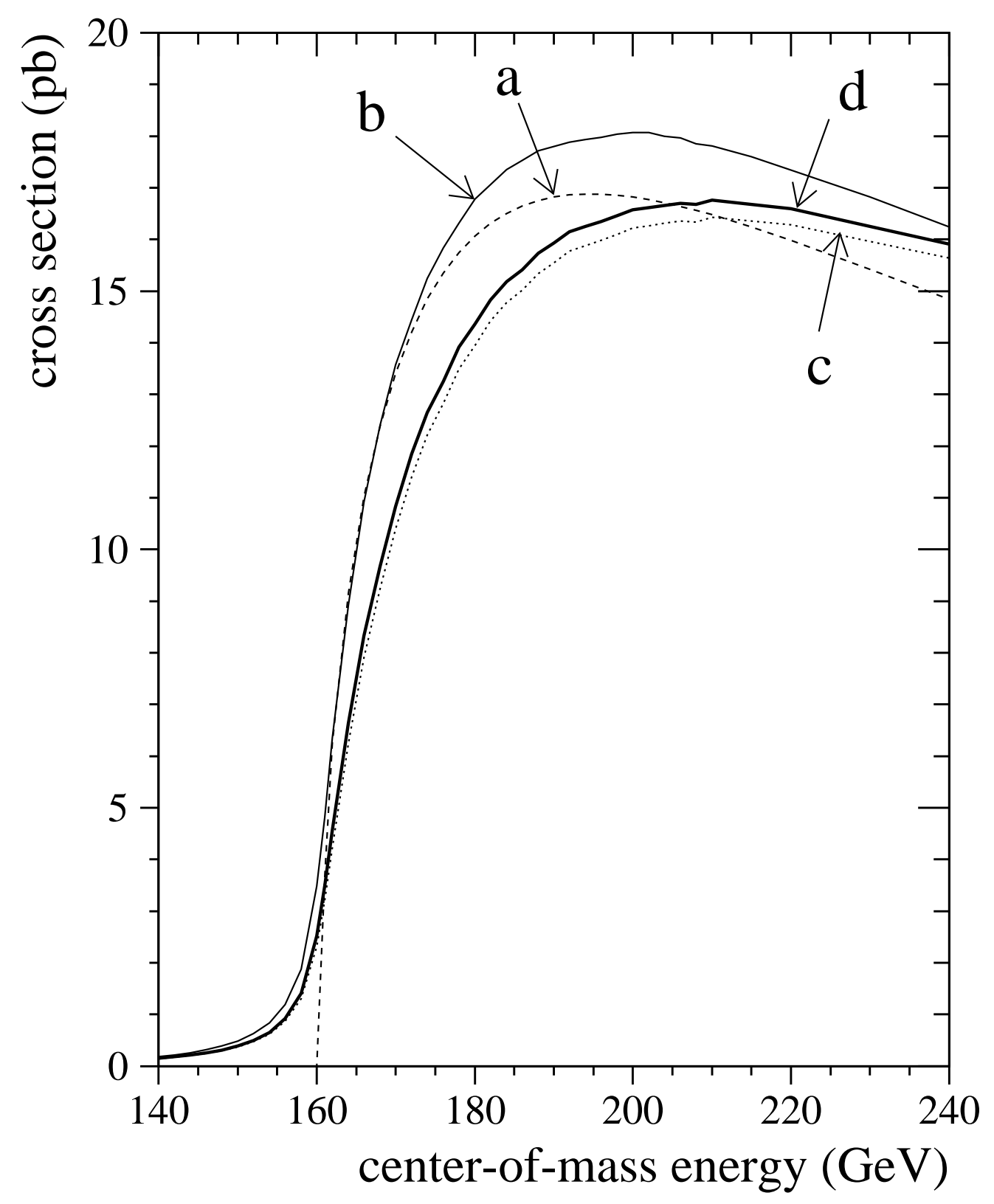

Figure 2: Total cross section as a function of the center-of-mass energy. Curve $a$ shows the Born level result, and $b$ shows the effect of the finite $W$ width. Curve $c$ shows how the cross section is reduced by ISR, and $d$, the final result, shows that the Coulomb singularity increases it by a couple of percent. 


\begin{tabular}{|c|cccc|}
\hline $\begin{array}{c}\sqrt{s} \\
(\mathrm{GeV})\end{array}$ & $a$ & $b$ & $c$ & $d$ \\
\hline 150 & 0 & $0.489 \pm 0.003$ & $0.369 \pm 0.003$ & $0.390 \pm 0.002$ \\
160 & 0 & $3.488 \pm 0.007$ & $2.316 \pm 0.005$ & $2.523 \pm 0.006$ \\
161 & 3.679 & $4.827 \pm 0.016$ & $3.210 \pm 0.008$ & $3.494 \pm 0.008$ \\
170 & 13.41 & $13.57 \pm 0.04$ & $10.40 \pm 0.02$ & $10.83 \pm 0.03$ \\
176 & 15.35 & $15.84 \pm 0.05$ & $12.83 \pm 0.03$ & $13.25 \pm 0.03$ \\
184 & 16.50 & $17.35 \pm 0.06$ & $14.78 \pm 0.03$ & $15.19 \pm 0.04$ \\
192 & 16.86 & $17.88 \pm 0.06$ & $15.77 \pm 0.04$ & $16.15 \pm 0.04$ \\
210 & 16.48 & $17.81 \pm 0.06$ & $16.42 \pm 0.04$ & $16.76 \pm 0.04$ \\
\hline
\end{tabular}

Table 1: Calculated total cross sections for selected $\sqrt{s}$, assuming $M_{\mathrm{W}}=80.25 \mathrm{GeV}$ and $\Gamma_{\mathrm{W}}=2.088 \mathrm{GeV}$. Column headings correspond to the curves in figure 2. Errors are statistical only.

the Coulomb singularity, and the mass-dependent width, one can observe indirect changes in the distributions of several physical observables as these corrections are included in the generator. For example, the invariant mass of fermion pairs is shown in figure 3, taken for $\sqrt{s}=175 \mathrm{GeV}$. The suppression of masses above $90 \mathrm{GeV}$ is evident in the top plot. The effects of ISR and Coulomb corrections are noticeable in the bottom plot, which shows the ratios of corrected to uncorrected distributions.

Initial-state radiation changes the angular distribution of the bosons in the lab frame, as shown in figure 4, causing it to be slightly less peaked in the forward direction. This would be important when constraining anomalous couplings. The change is mirrored in the angular distribution of individual fermions. Figure 5 illustrates this for the up-type fermion from the $\mathrm{W}^{+}$decay. A dramatic effect is seen in the momentum distribution of fermions, as seen in figure 6 . The slope of the distribution between the endpoints, which reflects the $V-A$ couplings as well as the $W$ polarisation, is greatly modified by initial-state radiation, as is the shape of the upper endpoint. These effects are not entirely removed when taking energy-independent quantities, such as the lab angle between fermions, or the ratio of fermion momenta, as shown in figure 7 .

\section{Comparisons with other Calculations}

We have compared our Monte Carlo event generator with other programs for four-fermion production, namely, GENTLE [16], EXCALIBUR [2], and PYTHIA [3]. GENTLE is a semi-analytical calculation of four-fermion processes through resonating $W$ pairs. It can provide some $d-$ ifferential distributions but not full event kinematics. EXCALIBUR is an event generator for any four-fermion final state, while PYTHIA is based on the JETSET package, and includes W-pair production as a distinct sub-generator. 

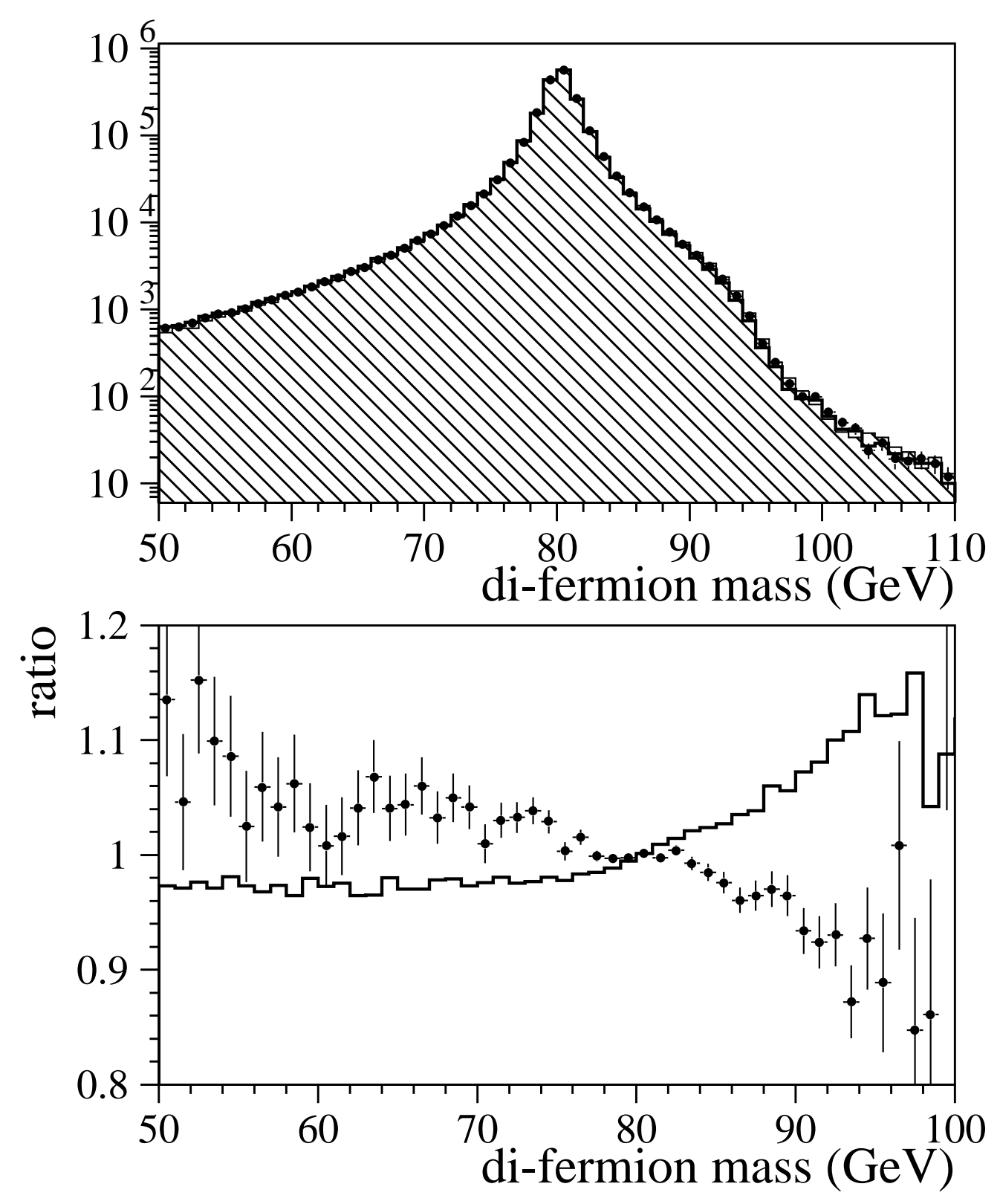

Figure 3: Invariant masses of pairs of fermions (two per event). For this and subsequent figures, in the top plot, the points represent the full calculation, the heavy line represents the calculation without Coulomb singularity, and the shaded histogram, the calculation without Coulomb singularity and without initial-state radiation. In the bottom plot, the solid line represents the ratio of the distributions for the calculation with Coulomb singularity over the full calculation, and the points, that of the calculation without Coulomb singularity or initial-state radiation and the full calculation. The error bars are due to Monte Carlo statistics. They are negligible for the solid line. $\sqrt{s}=175 \mathrm{GeV}$ 

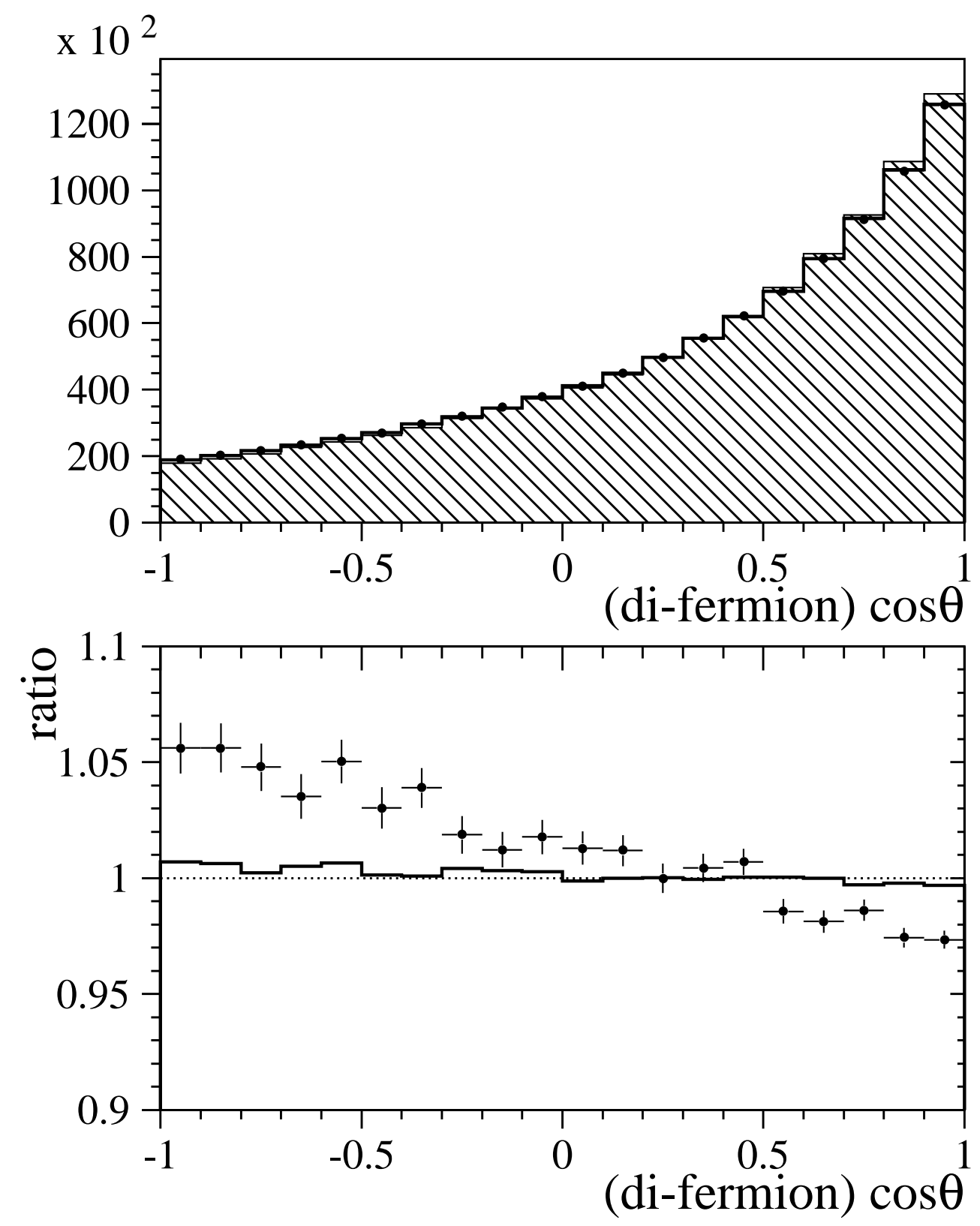

Figure 4: Distribution of the $\mathrm{W}^{-}$production angle in the lab frame. ( $\theta$ is the angle between the $\mathrm{W}^{-}$and the beam electron.) 

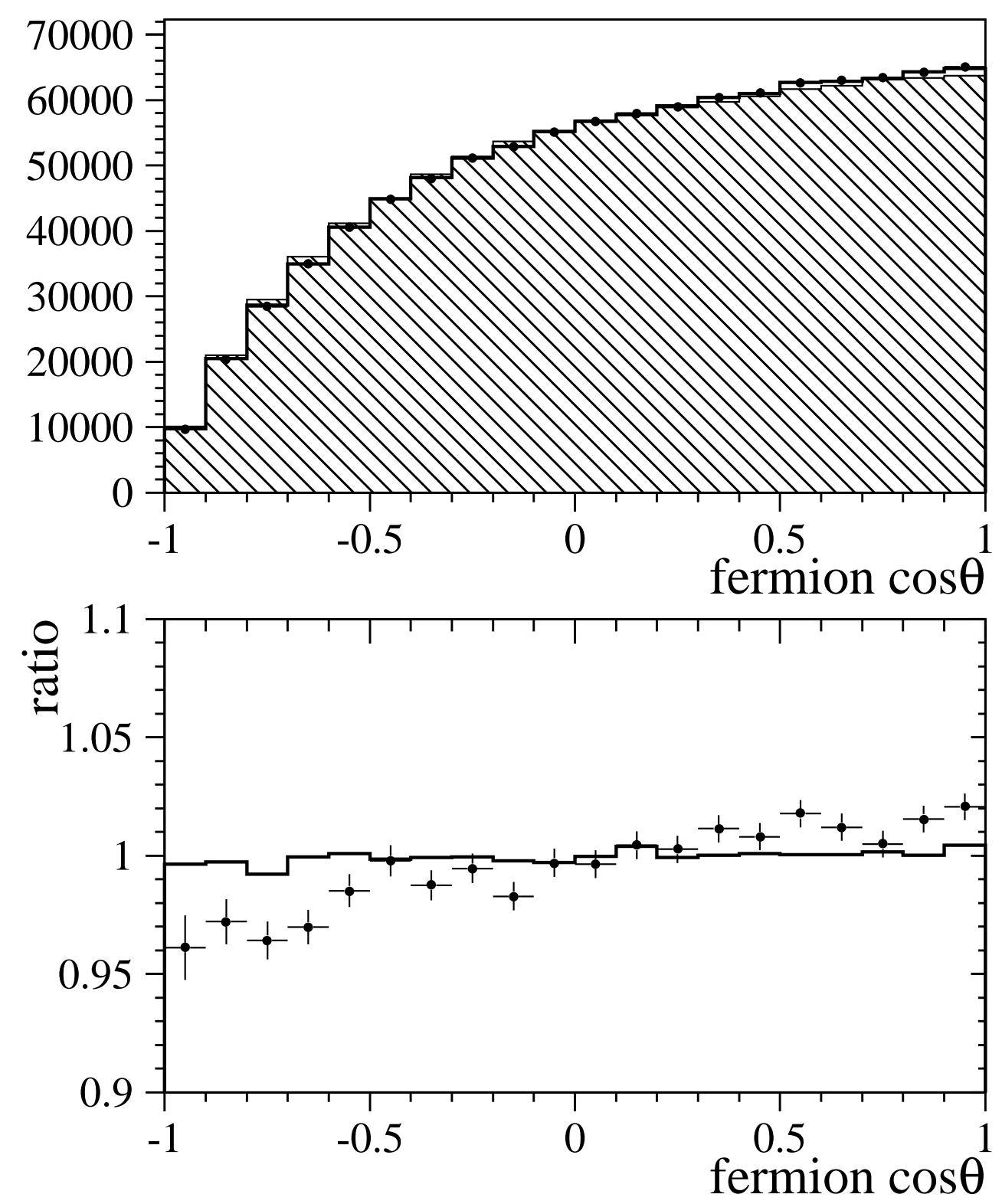

Figure 5: Distribution of the fermion production angle in the lab frame. For this plot, the up-type fermion from the $\mathrm{W}^{+}$has been selected. 

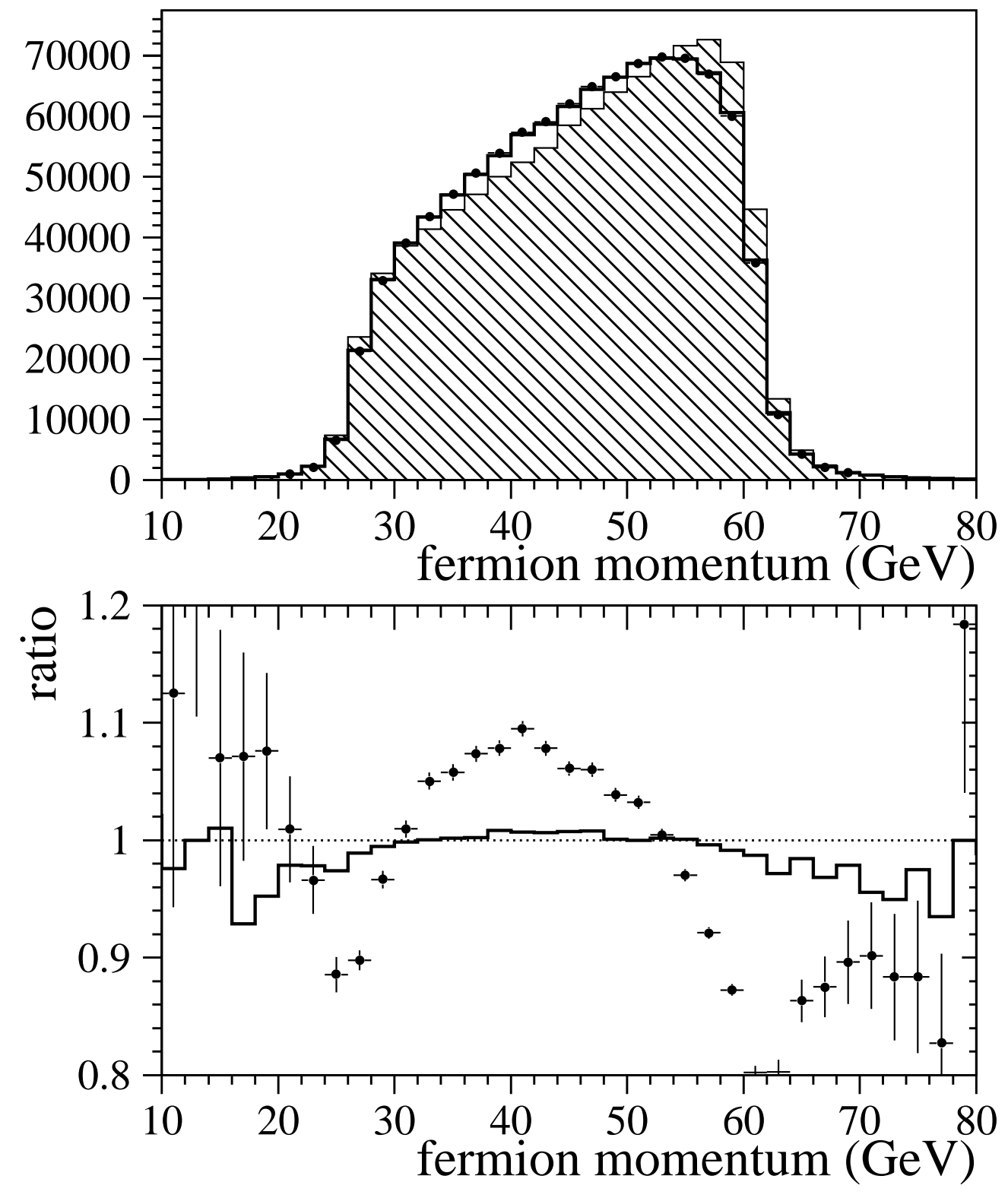

Figure 6: Fermion momentum distribution in the lab frame, shown for the anti-down-type fermion from the $\mathrm{W}^{+}$. 

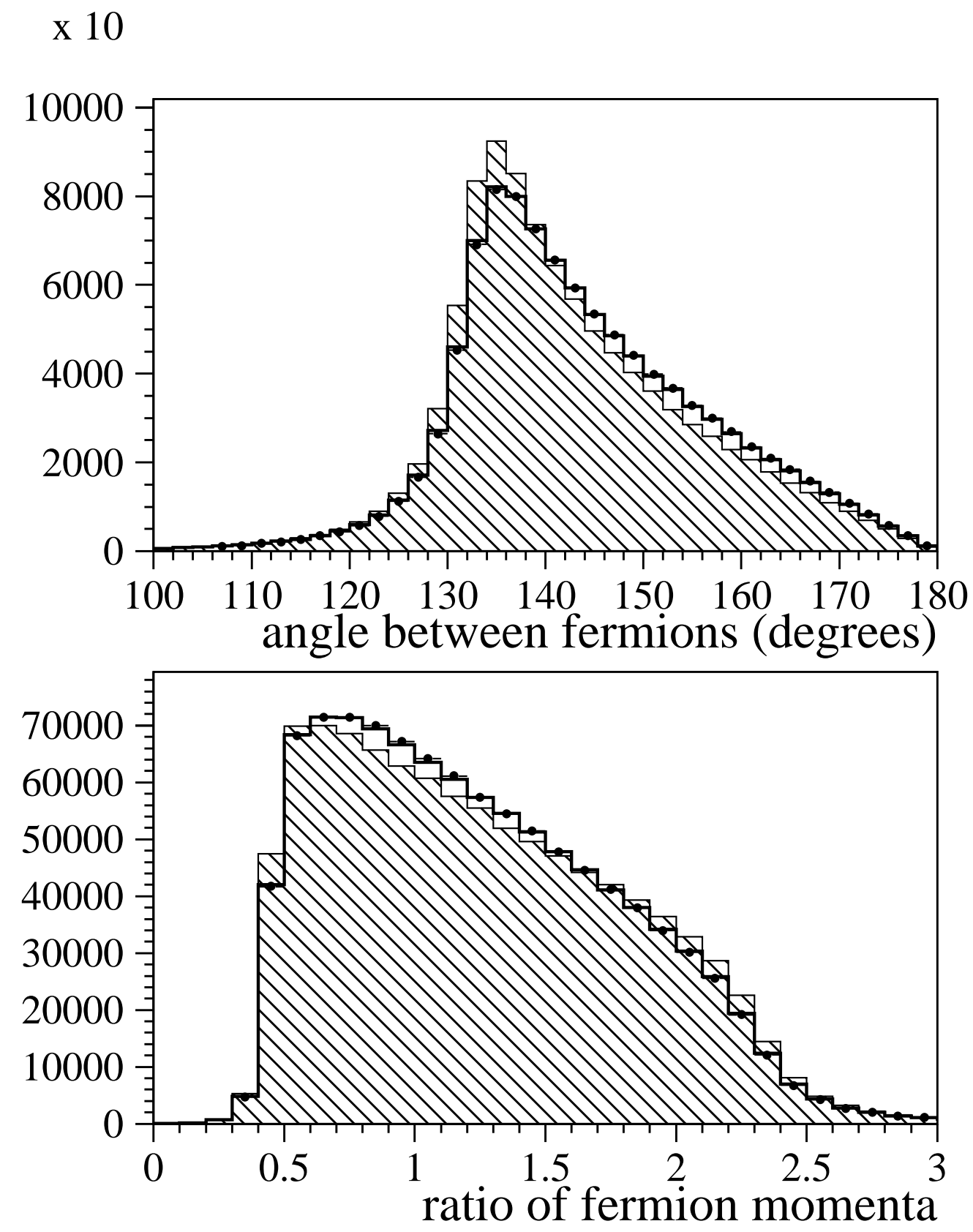

Figure 7: The lab angle between fermions is greatly effected by initial-state radiation, as shown in the top plot. The bottom plot shows the ratio of the fermion momenta. 


\begin{tabular}{|c|cc|cc|}
\hline \multirow{2}{*}{$\sqrt{s}(\mathrm{GeV})$} & \multicolumn{2}{|c|}{ cross section $(\mathrm{pb})$} & \multicolumn{2}{c|}{ mean photon } \\
\cline { 2 - 5 } & GENTLE & LPWW & GENTLE & LPWW \\
\hline 165 & $8.29 \pm 0.02$ & $8.31 \pm 0.01$ & $0.707 \pm 0.007$ & $0.710 \pm 0.007$ \\
170 & $11.62 \pm 0.03$ & $11.64 \pm 0.01$ & $0.984 \pm 0.009$ & $0.970 \pm 0.009$ \\
180 & $15.22 \pm 0.02$ & $15.18 \pm 0.01$ & $1.647 \pm 0.015$ & $1.626 \pm 0.015$ \\
190 & $16.74 \pm 0.03$ & $16.73 \pm 0.01$ & $2.347 \pm 0.022$ & $2.324 \pm 0.022$ \\
205 & $17.52 \pm 0.04$ & $17.43 \pm 0.02$ & $3.398 \pm 0.031$ & $3.348 \pm 0.031$ \\
\hline
\end{tabular}

Table 2: Comparison of the total WW cross section and mean energy loss as calculated by GENTLE and LPWW under comparable conditions.

\subsection{Comparison to GENTLE}

The comparison to GENTLE checks the WW-mediated part of the cross section together with the implementation of ISR. In order for the programs to be equivalent theoretically, we used the same prescription for the Coulomb singularity as used in GENTLE, and removed the contributions of ZZ diagrams. GENTLE was run with an option that uses the same ISR calculation as used in LPWW. It does not include $\alpha_{\mathrm{s}}$ corrections to the width, so we turned these off in LPWW. We used the effective couplings described above, but naturally, $\alpha_{\mathrm{QED}}=1 / 137$ for the ISR. The $W$ mass and width were $80.0 \mathrm{GeV}$ and $2.02 \mathrm{GeV}$.

A comparison of the total cross sections and mean energy loss due to ISR radiation is given in table 2. The energy loss is defined to be

$$
E_{\mathrm{ISR}}=\frac{\sqrt{s}}{2}\left(1-z_{1} z_{2}\right)
$$

where $z_{i}$ is the beam energy fraction after radiation.

The agreement between the two calculations is good. (See, for example, figure 8.) Using our evaluation of the Coulomb singularity increases the cross section by $0.3 \%$ at $170 \mathrm{GeV}$. Using the default ISR calculation in GENTLE, which is based in the 'current-split technique' [16], the cross section decreases by $2 \%$, but the mean energy loss changes by less than $10 \mathrm{MeV}$.

\subsection{Comparison to EXCALIBUR}

The comparison with EXCALIBUR checks the ZZ cross section and interference terms as well as the frequency of specific final states. The main difference between EXCALIBUR and LPWW is the inclusion of "background" diagrams (diagrams which include fewer than two resonating bosons) in EXCALIBUR. We have attempted to evaluate the importance of these diagrams to event samples selected in actual analyses (i.e., rather than to the total cross section).

Since ISR was checked with GENTLE, we turn it off for both programs. Both programs use mass-dependent widths, but EXCALIBUR lacks any account of the Coulomb singularity, so we 


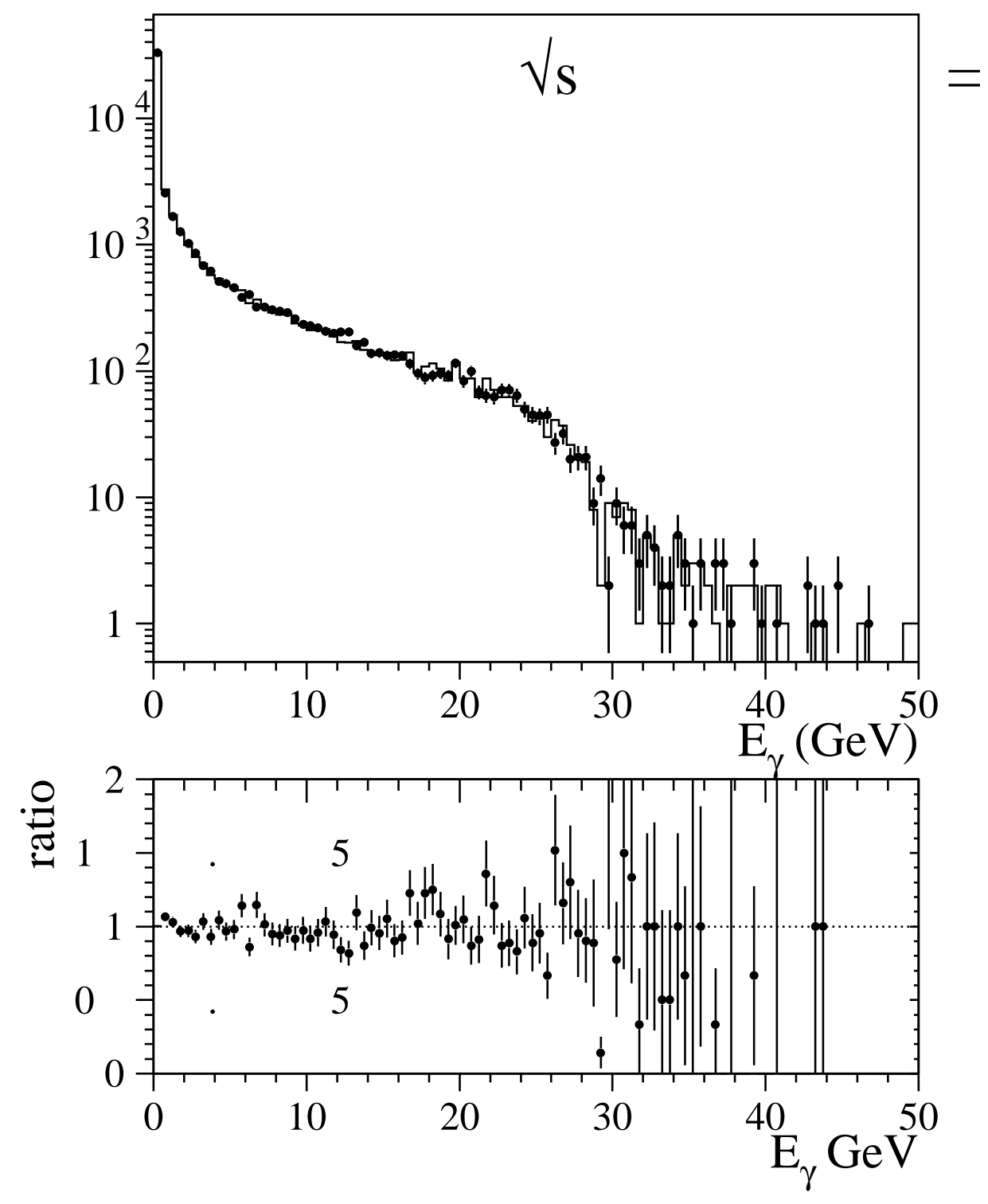

Figure 8: Energy of photons emitted in initial-state radiation. The points represent LPWW, and the solid line, GENTLE. Below the ratio of the distributions for LPWW over GENTLE is plotted. 
turned this off in LPWW. The effective couplings are taken into account in EXCALIBUR using a running $\alpha_{\mathrm{QED}}$, so we changed LPWW to use the same. For both programs we took $\alpha_{\mathrm{QED}}=$ $1 / 128$, so for a $W$ mass of $80.25 \mathrm{GeV}$, the width is $2.08 \mathrm{GeV}$.

The comparison of total cross sections is given in table 3 . The first state, $\mu^{-} \bar{\nu}_{\mu} \overline{\mathrm{d}} u$, is the simplest, as there is no contribution from Z's. After switching off the background diagrams in EXCALIBUR, the agreement is perfect. The next state, $\mu^{-} \mu^{+} \bar{u} u$, can come only from noninterfering neutral bosons. For EXCALIBUR, we turned off again the background diagrams, and obtained agreement again, both above the ZZ threshold, and just below it. To test the interference between the Z's which occurs when all four fermions have the same colour and flavour, we compared the ūūu final state, and found agreement. On the basis of these comparisons, we conclude that the matrix element and phase space part of LPWW is correct.

Four-fermion event samples collected at LEP2 will contain contributions from processes involving only one $\mathrm{W}$ or $\mathrm{Z}$ boson, with possibly one or two virtual photons and gluons, as discussed in [2]. LPWW does not take any of these contributions into account. It may still be reasonably accurate, however, since many of these events will be excluded by analysis requirements. In order to quantify this situation, we calculated the effective cross section for events passing the following experimental 'cuts':

- All fermions must make an angle $\theta$ with the beam such that $|\cos \theta|<0.9$.

- All fermions must have at least $10 \mathrm{GeV}$ energy.

- The invariant mass of all six pairs of fermions must be at least $10 \mathrm{GeV}$.

The first cut ensures that the jet (or lepton) can be well measured by the apparatus, and the second and third cuts ensure that the jets are distinct. Depending on $\sqrt{s}, 60-70 \%$ of the events generated by LPWW satisfy these requirements.

Differences between the predictions of LPWW and EXCALIBUR can be seen for the state $\mathrm{d} \overline{\mathrm{u}} \overline{\mathrm{d}} \mathrm{u}$, especially near threshold. At $\sqrt{s}=161 \mathrm{GeV}$, LPWW predicts an accepted cross section which is $2.7 \%$ too low, because it lacks the background diagrams. At higher energies, the discrepancy is smaller. The $\mathrm{e}^{-} \bar{\nu}_{\mathrm{e}} \overline{\mathrm{d}} u$ final state is expected to be worse, since the presence of an electron in the final state means there are many more background diagrams calculated by EXCALIBUR. As expected, there are significant differences between the two calculations. The difference reaches $6 \%$ at $161 \mathrm{GeV}$, while it is below $2 \%$ at higher energies. It should be noted that these and analogous final states represent a small fraction of the total, so the error on the total cross section (i.e., applying the cuts but summing over all fermion flavours) will be much smaller.

\subsection{Comparison to PYTHIA}

The GENTLE and EXCALIBUR generators do not address hadronisation, but PYTHIA [3], based on the string model, is expected to simulate hadronisation well. A comparison of the charged 


\begin{tabular}{|cc|ccl|}
\hline $\begin{array}{c}\text { final } \\
\text { state }\end{array}$ & $\sqrt{s}(\mathrm{GeV})$ & \multicolumn{3}{|c|}{ cross section $(\mathrm{fb})$} \\
\hline$\mu^{-} \bar{\nu}_{\mu} \mathrm{d} u$ & 160 & $120.2 \pm 0.4$ & $120.4 \pm 0.3$ & total; WW only \\
& 161 & $166.6 \pm 0.5$ & $167.1 \pm 0.4$ & \\
& 165 & $348.9 \pm 0.8$ & $349.9 \pm 0.9$ & \\
& 175 & $555.2 \pm 1.2$ & $556.4 \pm 1.5$ & \\
& 190 & $640.8 \pm 1.5$ & $642.2 \pm 1.7$ & \\
\hline$\mu^{-} \mu^{+} \bar{u} u$ & 180 & $1.284 \pm 0.002$ & $1.282 \pm 0.002$ & total; ZZ only \\
& 190 & $6.976 \pm 0.006$ & $6.973 \pm 0.002$ & \\
\hline $\mathrm{u} \bar{u} \bar{u} u$ & 190 & $11.87 \pm 0.01$ & $11.88 \pm 0.01$ & total; ZZ with interference \\
\hline $\mathrm{d} \bar{u} \mathrm{~d} u$ & 161 & $363.5 \pm 1.4$ & $353.9 \pm 1.1$ & after cuts; WW, ZZ, background \\
& 175 & $1110 \pm 4$ & $1103 \pm 3$ & \\
& 190 & $1243 \pm 4$ & $1229 \pm 3$ & \\
\hline $\mathrm{e}^{-} \bar{\nu}_{\mathrm{e}} \overline{\mathrm{d}} \mathrm{u} u$ & 161 & $111.8 \pm 0.4$ & $118.6 \pm 0.4$ & after cuts; WW, background \\
& 175 & $369.3 \pm 1.2$ & $367.1 \pm 1.1$ & \\
& 190 & $412.9 \pm 1.4$ & $404.1 \pm 1.2$ & \\
\hline
\end{tabular}

Table 3: Comparison of the total cross section (in $f b$ ) as calculated by EXCALIBUR and LPWW under comparable conditions. See the text for a description of the cuts.

multiplicity, the total visible energy, and the event thrust as simulated using LPWW and PYTHIA tests our implementation of gluon radiation, hadronisation and decays. A cutoff of $0.15 \mathrm{GeV}$ for the transverse momentum of charged tracks was imposed, as well as momentum thresholds of $0.25 \mathrm{GeV}$ and $0.5 \mathrm{GeV}$ for photons and neutral hadrons, respectively. Particles with $|\cos \theta|>0.95$ were excluded. The comparison at $\sqrt{s}=176 \mathrm{GeV}$, displayed in figure 9 , shows that the agreement is good for all distributions.

\section{Summary}

We have presented a practical Monte Carlo event generator for four-fermion processes through two resonating massive bosons. The tree level calculation is complete, including naturally the effect of the finite width of W's and Z's and the full transmission of spin information from initial to final state.

The main radiative corrections effects are included. Initial state radiation, using structure functions containing up to second order leading-logarithms plus soft-photon exponentiation. Final state radiation in the second order leading-logarithm approximation, using the PHOTOS[10] package. The Improved Born Approximation is used to include most electroweak corrections. Coulomb effects are also taken into account. Gluon radiation, hadronisation and decays are taken care of by the JETSET package [5]. 

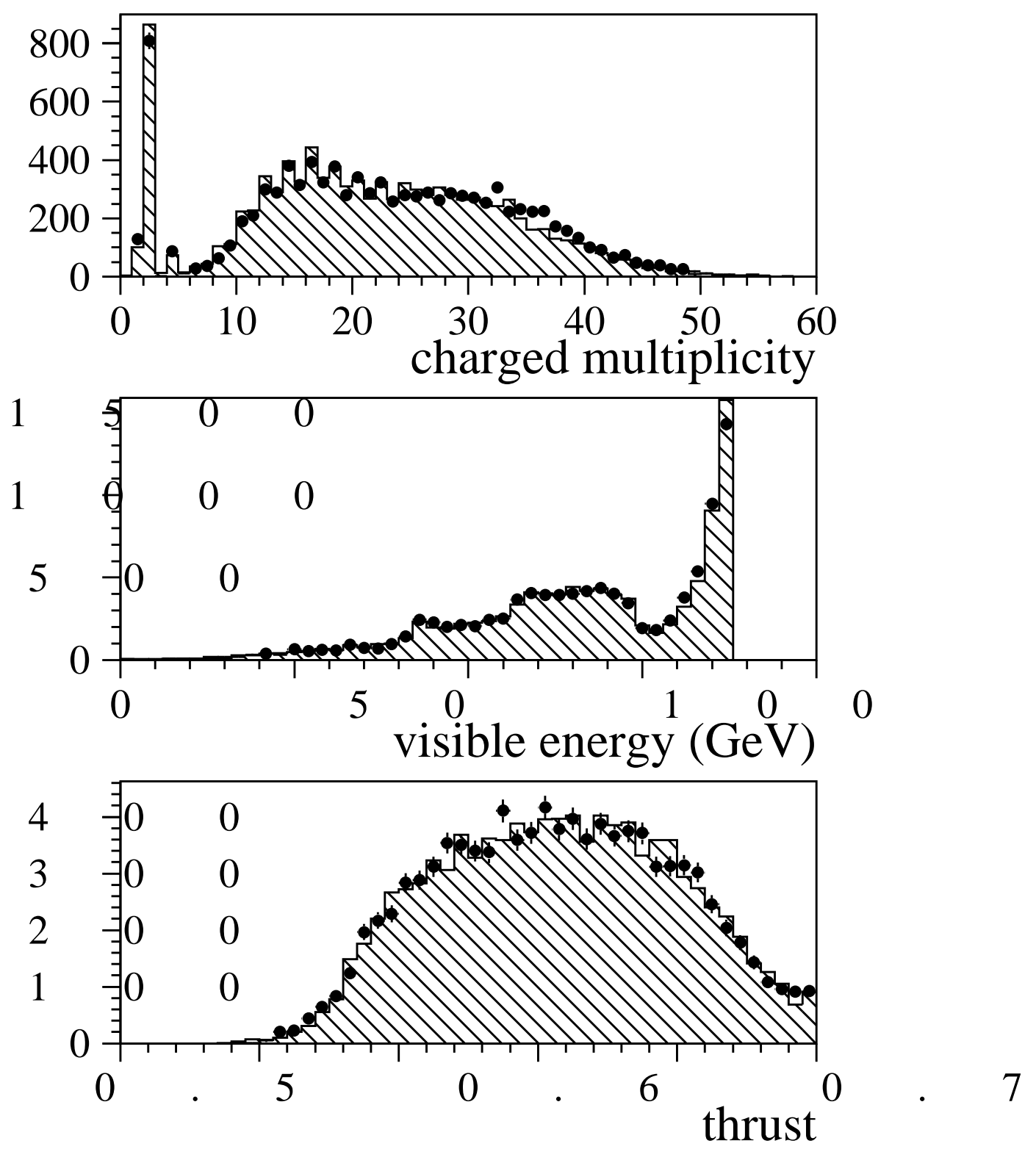

0

Figure 9: Total charged multiplicity, visible energy, and event thrust for the PYTHIA (shaded histogram) and LPWW (points) generators. 
The generator has been thoroughly compared with other existing calculations and excellent agreement has been found. The resulting program (LPWW) is well suited for experimental studies in preparation for physics at LEP2.

The effects of initial state radiation and the Coulomb singularity are large, and corrections are necessary for an experimental understanding of $W$ pair production, as shown by our studies. In contrast, once very minimal experimental selection cuts are applied to the generated events, background diagrams have only a moderate influence on the cross sections, especially at energies above the threshold region.

\section{Acknowledgements}

We like to thank A. Olshevskii for making the GENTLE program available to us for the comparisons, and R. Kleiss for useful comments. 


\section{References}

[1] H. Anlauf et al, Comput. Phys. Commun. 79 (1994) 487;

G. J. van Oldenborgh, P. J. Franzini and A. Borelli, Comput. Phys. Commun. 83 (1994) 14 ;

G. Montagna, O. Nicrosini, G. Passarino and F. Piccinini, Phys. Lett. B348 (1995) 178.

[2] F. A. Berends, R. Pittau and R. Kleiss, Nucl. Phys. B424 (1994) 308.

[3] T. Sjöstrand, CERN-TH 7112/93.

[4] R. Kleiss, Monte Carlo program LEPWW, unpublished.

[5] T. Sjöstrand, Comput. Phys. Commun. 39 (1986) 347;

T. Sjöstrand and M. Bengtsson, Comput. Phys. Commun. 43 (1987) 367;

H.-U. Bengtsson and T. Sjöstrand, Comput. Phys. Commun. 46 (1987) 43 (and references contained therein).

[6] R. Kleiss and W. J. Stirling, Nucl. Phys. B262 (1985) 235.

[7] F. A. Berends, P. H. Daverveldt, R. Kleiss, Nucl. Phys. B253 (1985) 441.

[8] E. A. Kuraev, V. S. Fadin, Sov. J. Nucl. Phys. 41 (1985) 466.

[9] O. Nicrosini, L. Trentadue, Phys. Lett. B196 (1987) 551.

[10] E. Barberio and Z. Was, CERN-TH/7033-93.

[11] F. A. Berends and R. Kleiss, Z. Phys. C27 (1985) 365.

[12] A. Denner, in Proceedings of the 28th Rencontres de Moriond: Electroweak Interactions and Unified Theories, Les Arcs (France) 1993. Ed. J. Tran Thanh Van, Editions Frontières, Gif-sur-Yvette (France), 1994, pp. 433-440.

[13] See, for instance, G. Gustafson, U. Pettersson, P. M. Zerwas, Phys. Lett. B209 (1988) 90.

[14] L. Lonnblad, T. Sjöstrand, Phys. Lett. B351 (1995) 293.

[15] V. S. Fadin, V. A. Khoze, A. D. Martin and A. Chapovskii, DTP-94-116.

[16] D. Bardin et al, CERN-TH 7295/94. 